\title{
The identification of ketotifen as a novel cardioprotective agent in patients undergoing anthracyclines chemotherapy
}

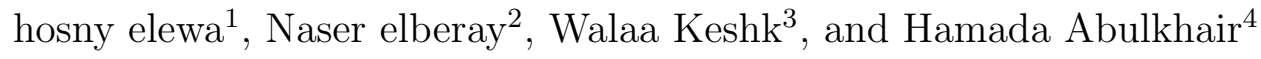 \\ ${ }^{1}$ Horus University \\ ${ }^{2}$ Menoufia University \\ ${ }^{3}$ Tanta University \\ ${ }^{4}$ Al-Azhar University
}

February 8, 2021

\begin{abstract}
Objective: The present study aimed to investigate the possible cardioprotective effects of ketotifen and to assess its activity as an iron-chelating agent in patients receiving anthracyclines for the treatment of breast cancer. Patients \& Methods: This was a randomized, prospective, controlled clinical trial. One hundred eleven eligible patients with breast cancer (age range, 30-60 years) were scheduled to receive anthracyclines chemotherapy. The patients were divided into two groups: Patients $(\mathrm{n}=56)$ assigned to the ketotifen group received ketotifen $1 \mathrm{mg}$ three times daily for six consecutive cycles of treatment, and patients assigned to the control group $(\mathrm{n}=55)$ without ketotifen treatment. The echocardiogram for each patient was recorded two times at baseline and the end of the study. As well, blood samples were collected from all patients. Results: The findings showed a statistically significant reduction in the mean serum levels of common cardiotoxicity accompanied biomarkers in the ketotifen group compared with the control group (P [?] 0.05). The mean serum levels of total iron-binding capacity were significantly elevated in the ketotifen group ( $\mathrm{P}$ [?] 0.001). There was a direct correlation between the mean serum levels of iron and that of lactate dehydrogenase $(\mathrm{LDH})(\mathrm{r}=+0.79)$. On the other hand, there were indirect correlations between mean serum levels of $\mathrm{LDH}$ and both the percentage of ejection fraction and the total iron-binding capacity $(\mathrm{r}=-0.69$ and -0.697 , respectively). Conclusion: Oral administration of ketotifen appears to be efficient and safe as a novel cardioprotective agent for the prevention of anthracyclines induced cardiotoxicity. Additionally, ketotifen suggested a beneficial effect in iron overload inducing diseases such as COVID-19.
\end{abstract}

\section{Hosted file}

IJCP MS.pdf available at https://authorea.com/users/387711/articles/507848-theidentification-of-ketotifen-as-a-novel-cardioprotective-agent-in-patients-undergoinganthracyclines-chemotherapy

\section{Hosted file}

List of Figures, Charts, and Tables.pdf available at https://authorea.com/users/387711/ articles/507848-the-identification-of-ketotifen-as-a-novel-cardioprotective-agent-inpatients-undergoing-anthracyclines-chemotherapy 